\title{
Anion exchange Resin Based Porous Carbon Spheres for the Catalytic Dehydrochlorination of Dichloroethane
}

Jing Wang, ${ }^{a, b}$ Sisi Fan, ${ }^{a}$ Hongmin Duan, ${ }^{a}$ Jinming $X u,{ }^{* a}$ and Yanqiang Huang, ${ }^{* a}$

${ }^{a}$ CAS Key Laboratory of Science and Technology on Applied Catalysis, Dalian

Institute of Chemical Physics, Chinese Academy of Sciences, Dalian 116023,

Liaoning, China.

bUniversity of Chinese Academy of Sciences, Beijing 100049, China.

*Corresponding author: xujm@dicp.ac.cn (J. Xu), yqhuang@dicp.ac.cn (Y. Huang) 


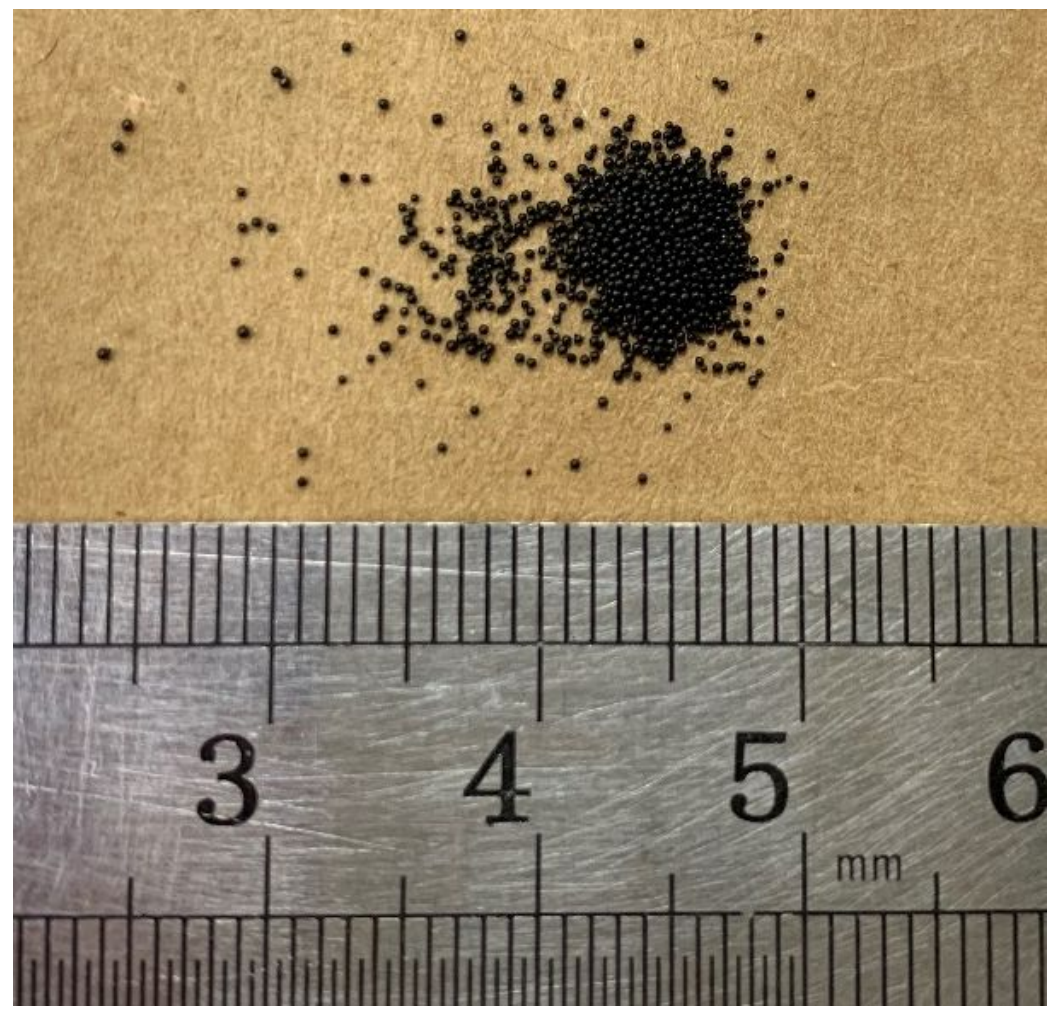

Figure S1 Photograph of carbon spheres 900-900-RC produced from resin spheres. 


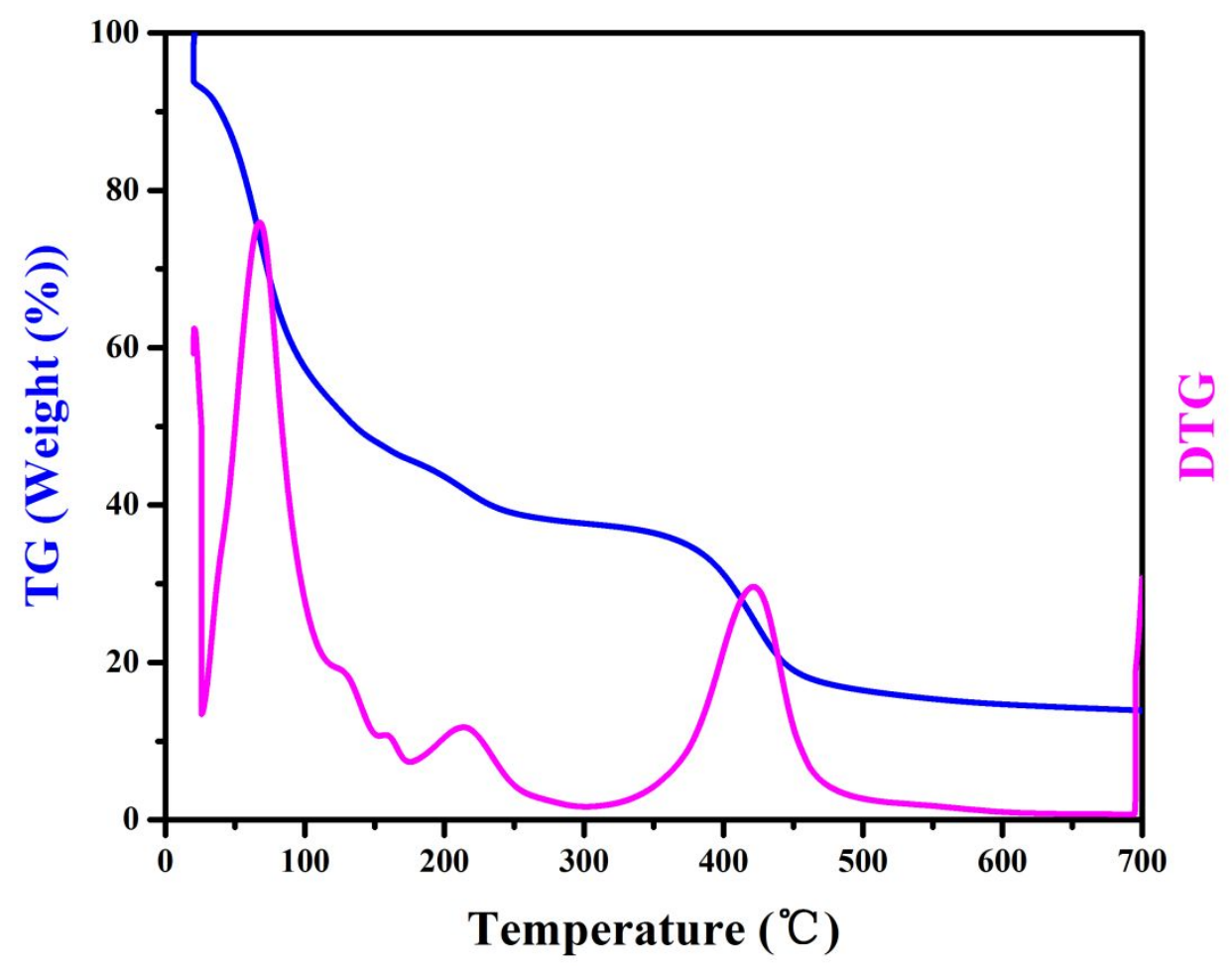

Figure S2 Thermogravimetric analysis during the pyrolysis process of anion exchanged resin under Ar atmosphere. 


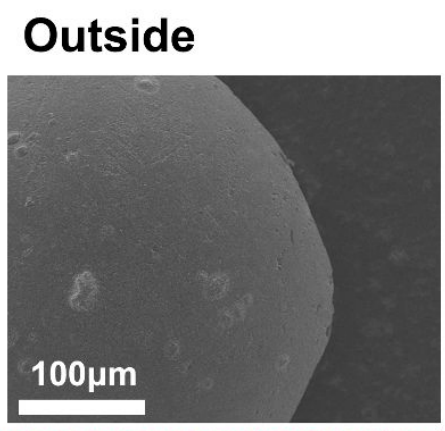

\section{Inside}

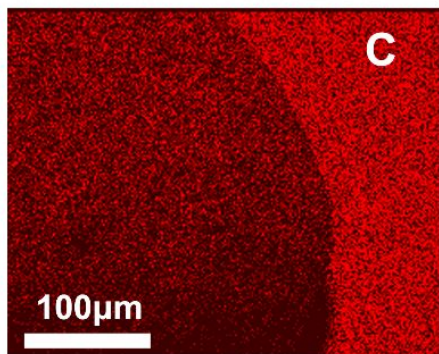

$100 \mu \mathrm{m}$
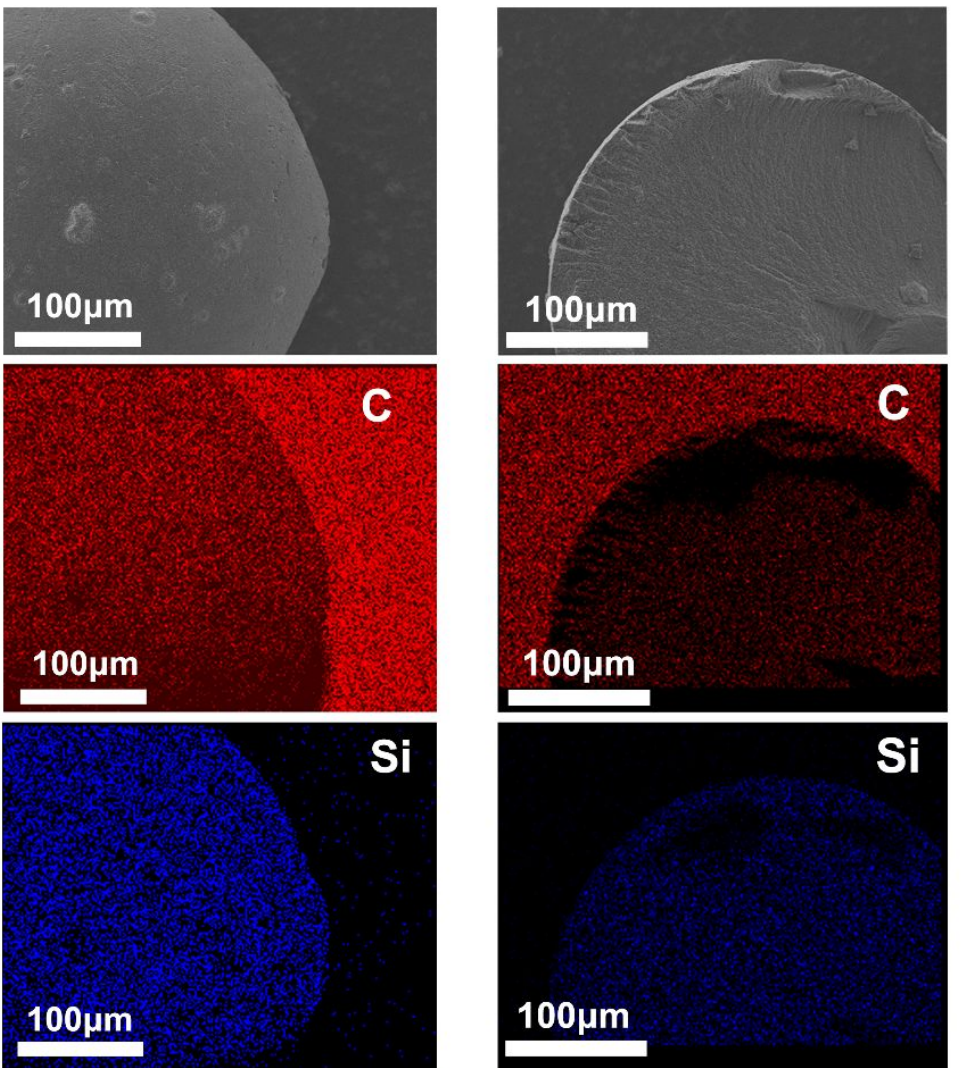

$100 \mu \mathrm{m}$

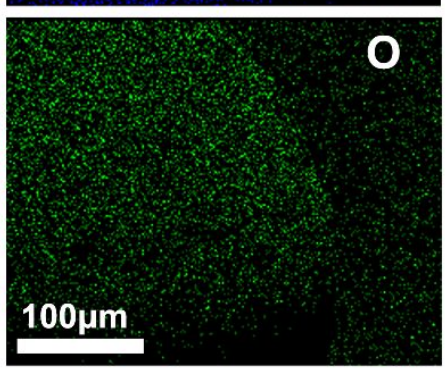

O

$100 \mathrm{um}$

Figure S3 The elemental distribution mapping of $\mathrm{C}, \mathrm{Si}, \mathrm{O}$ in the carbon and silica composite spheres. 


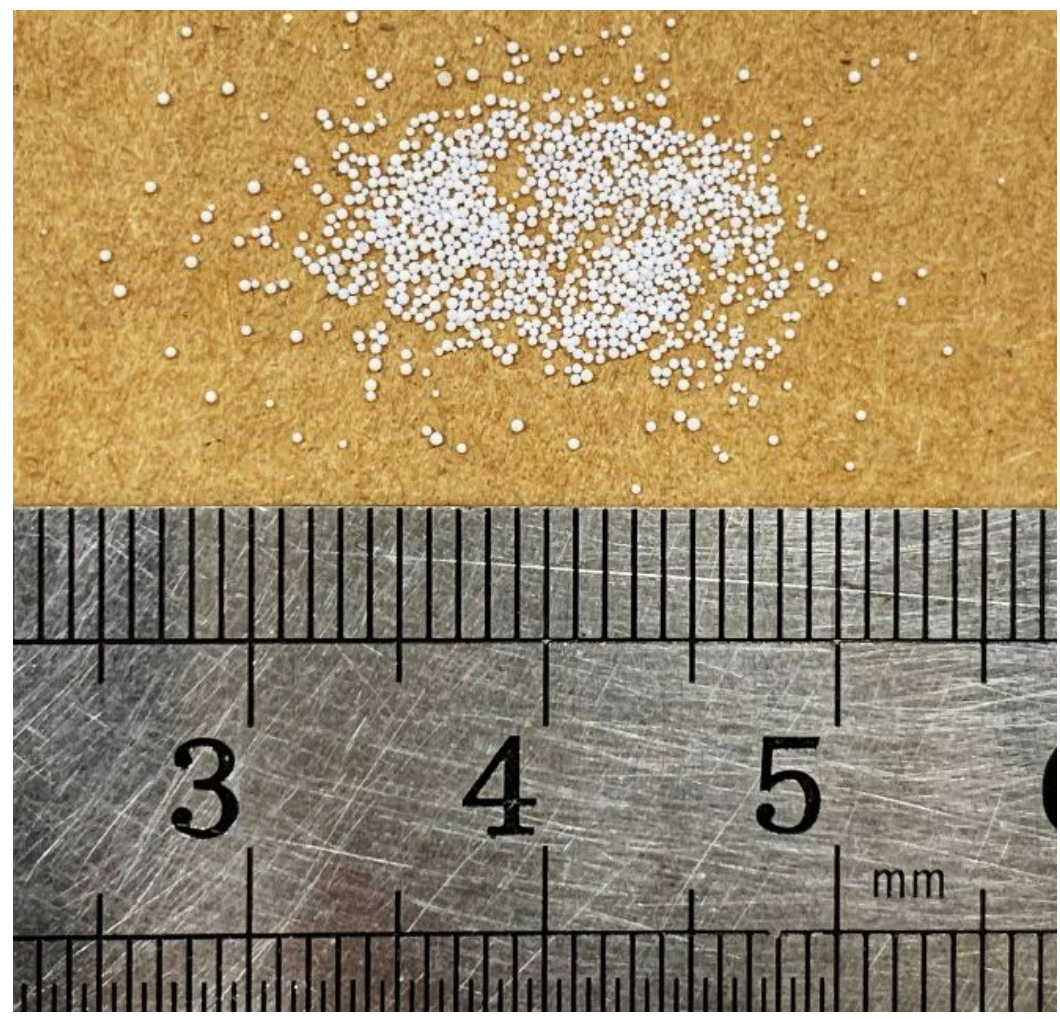

Figure S4 Photograph of $\mathrm{SiO}_{2}$ spheres (900-900-RS) produced from carbon and silica composite spheres, associated with 900-900-RC. 


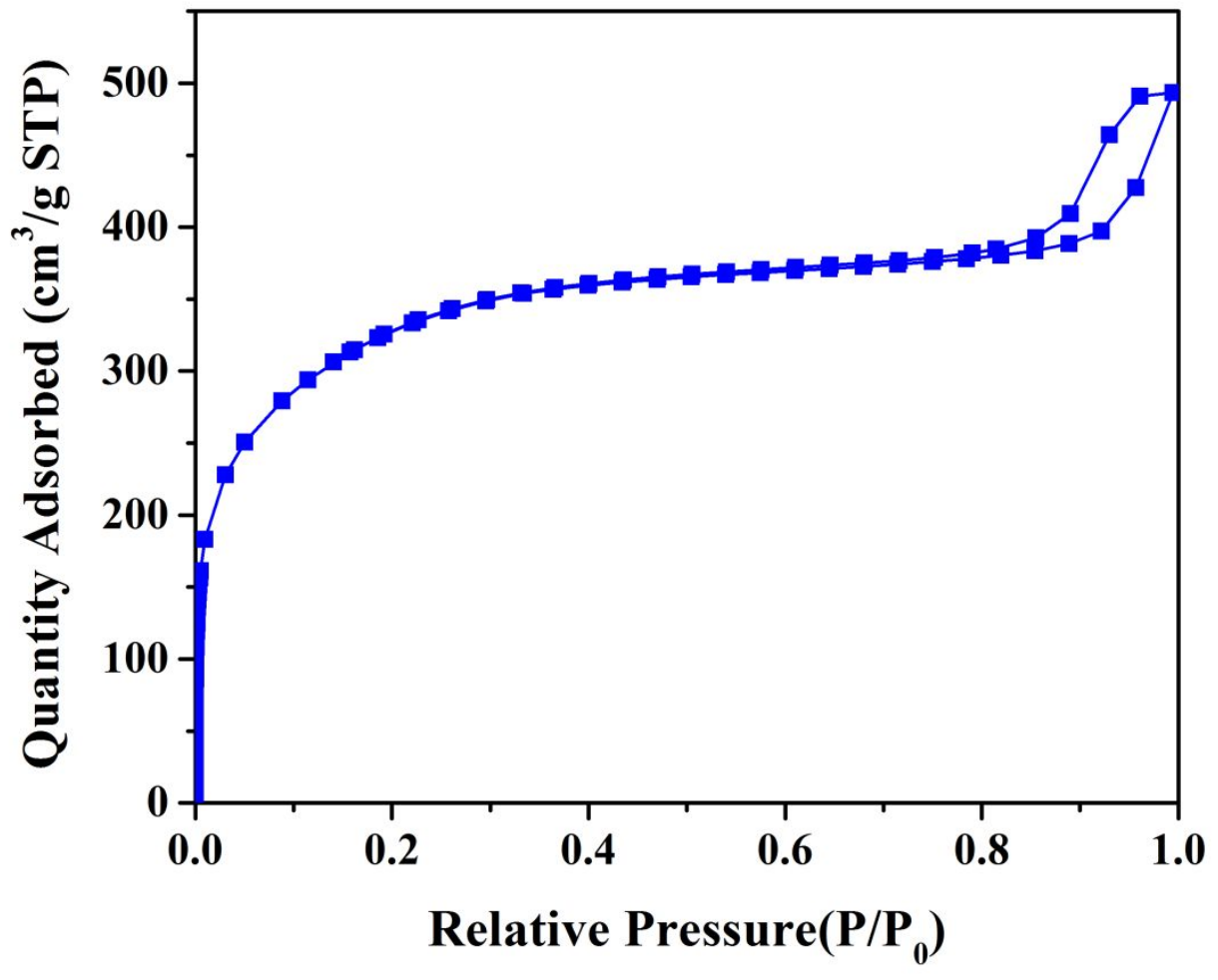

Figure S5 Nitrogen adsorption-desorption isotherm for $\mathrm{SiO}_{2}$ sphere (900-900-RS). 


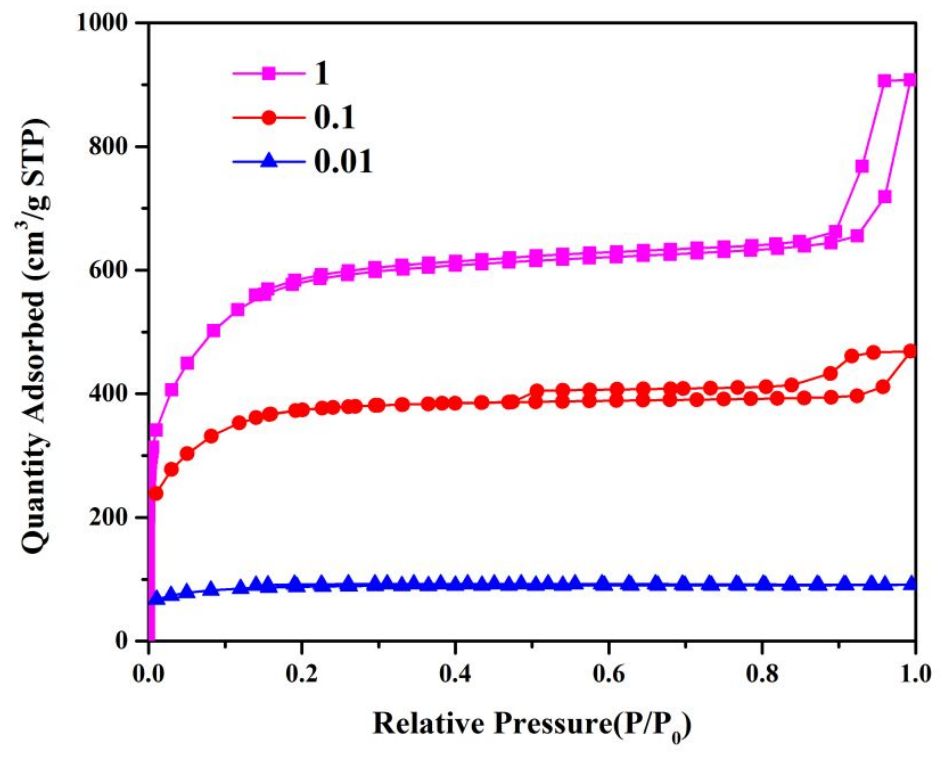

Figure S6 Nitrogen adsorption-desorption isotherms for carbon spheres made by different content of silicate 

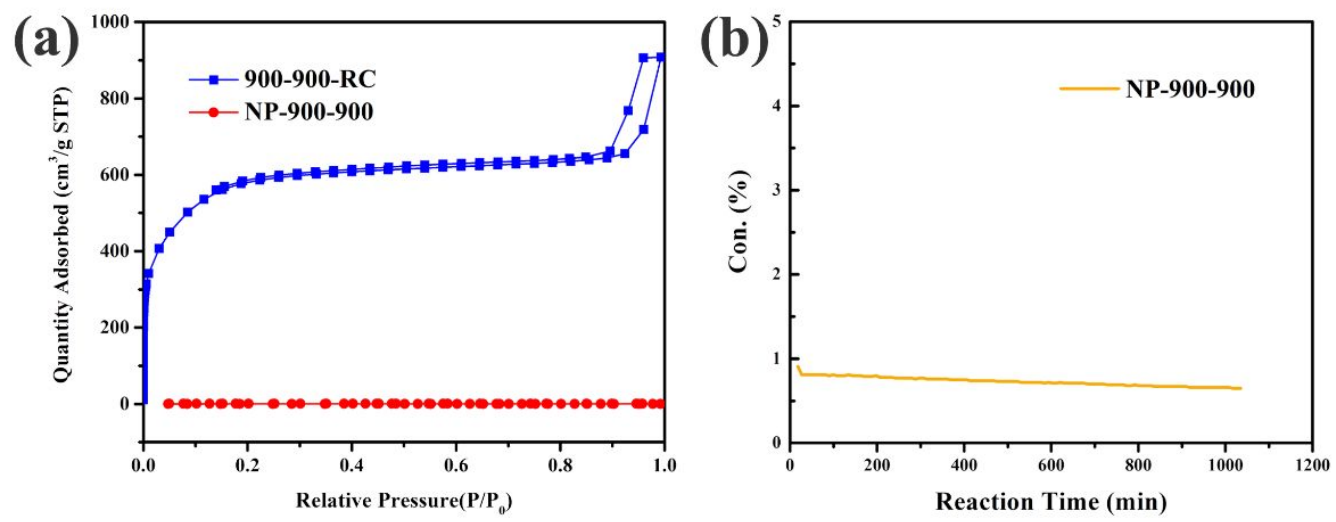

Figure S7 (a) $\mathrm{N}_{2}$ physical adsorption-desorption isotherms of 900-900-RC and NP900-900 (b) Catalytic performance of NP-900-900 (Reaction Conditions: $300{ }^{\circ} \mathrm{C}$ ). 


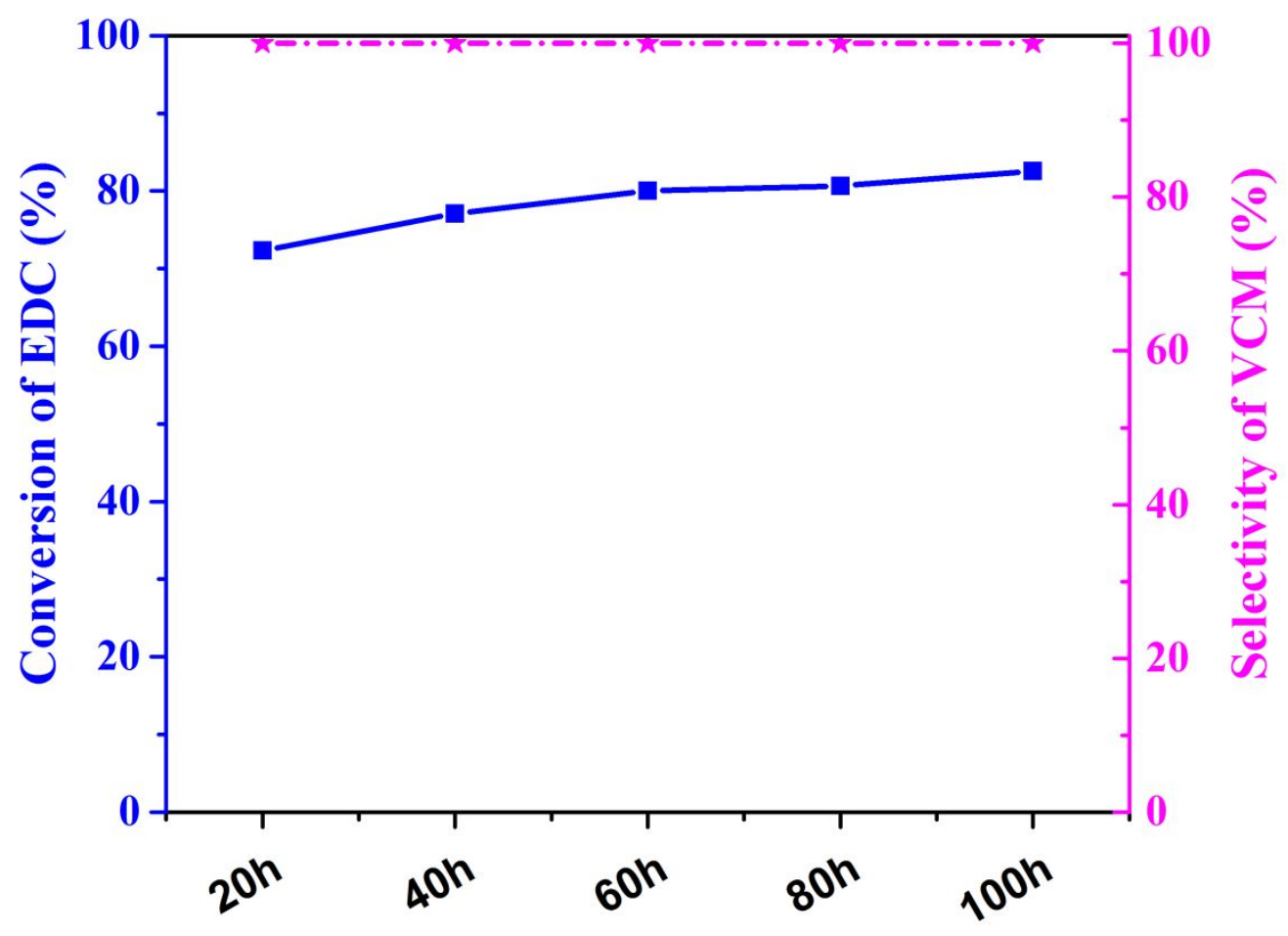

Figure S8 Catalytic stability performance of 900-900 (Reaction Conditions: $280{ }^{\circ} \mathrm{C}$ ). 

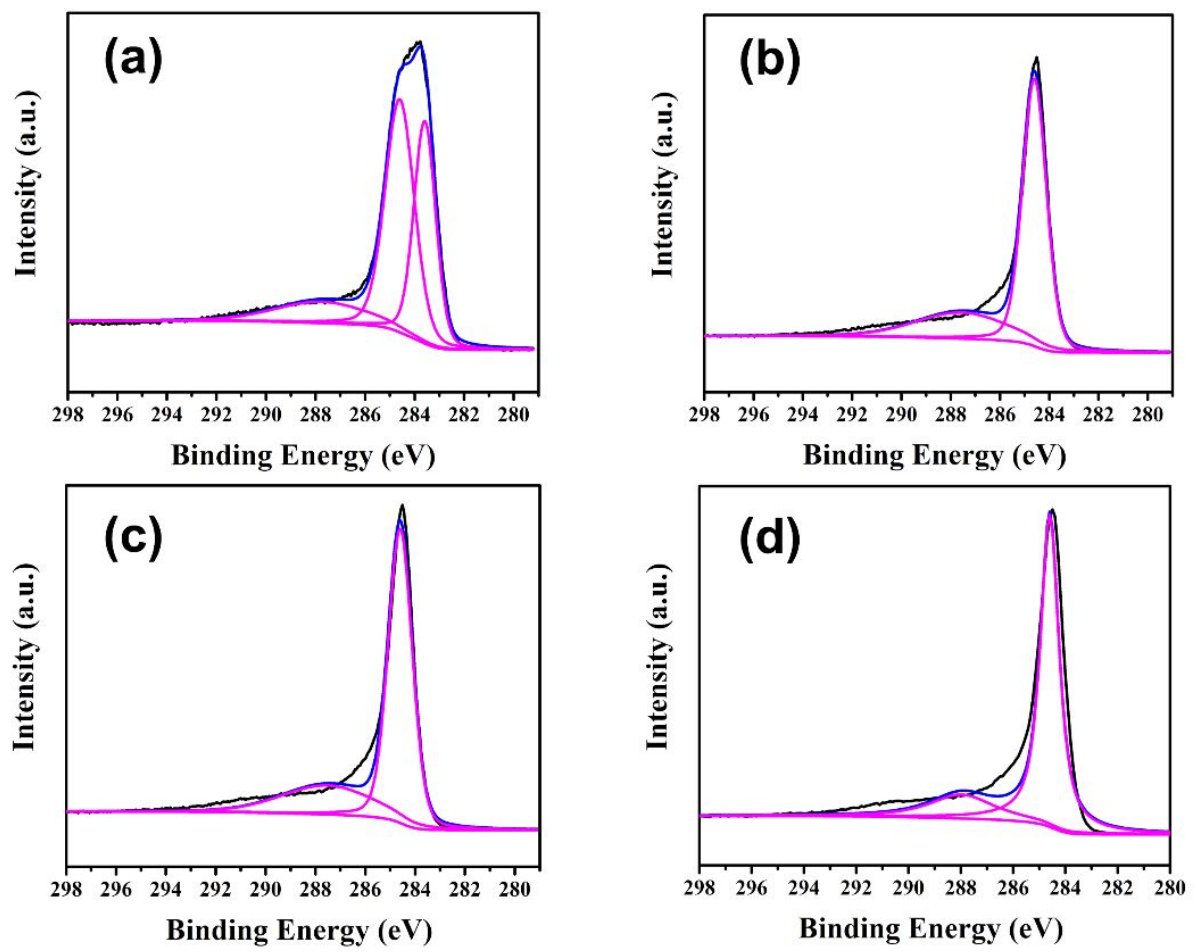

Figure S9 The high-resolution C1s spectra of (a) 600-900-RC catalysts, (b) 700-900RC catalysts, (c) 800-900-RC catalysts and (d) 900-900-RC catalysts. 

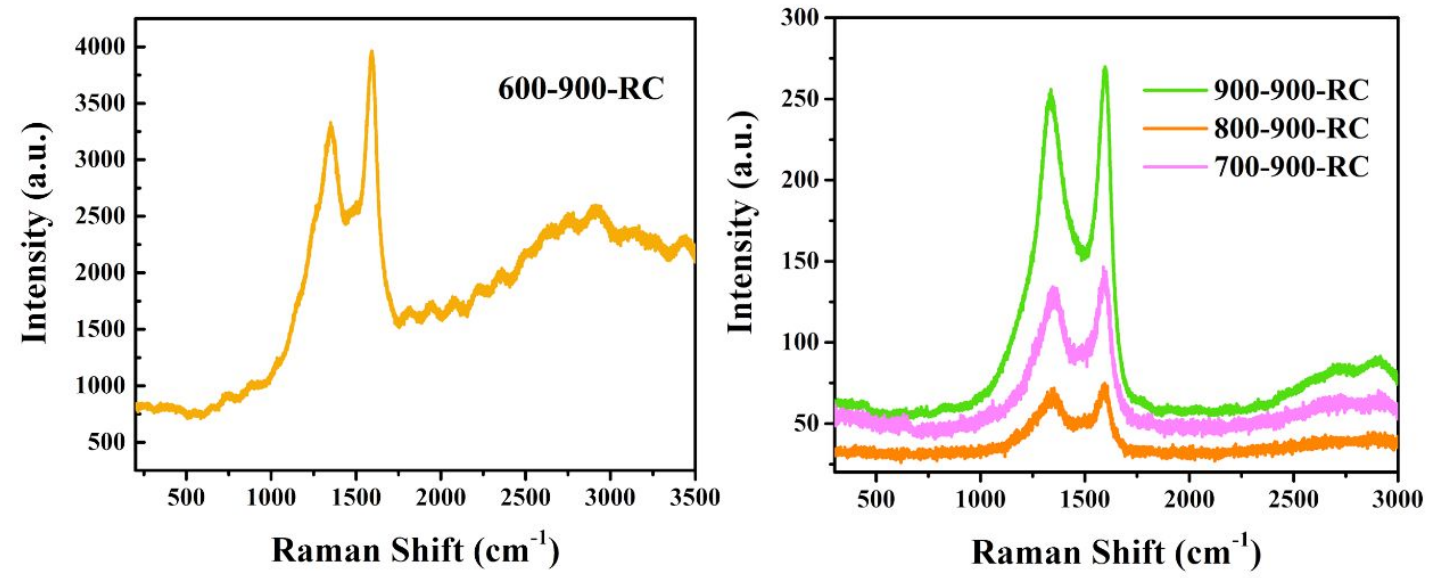

Figure S10 Raman spectrum of T1-900-RC. 
<smiles>C1=CN=C2C(=CCC3=C2C2=CCC=CC2=C3)C=C1</smiles>

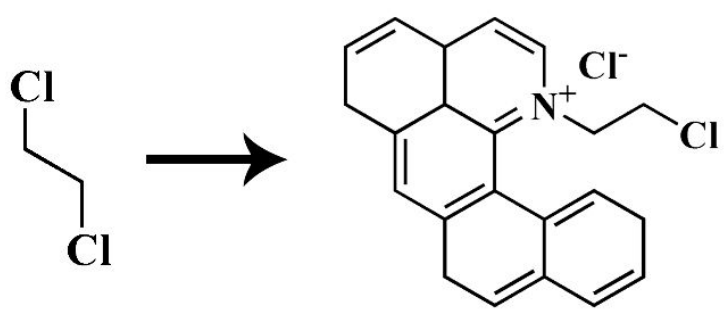

Figure S11 Schematic illustration of the pyridinic nitrogen species reaction with EDC 
Table S1 Raman results $\left(\mathrm{I}_{\mathrm{D}} / \mathrm{I}_{\mathrm{G}}\right)$ of $600-900-\mathrm{RC}, 700-900-\mathrm{RC}, 800-900-\mathrm{RC}$ and $900-$ 900-RC.

\begin{tabular}{c|cccc}
\hline Samples & $600-900-\mathrm{RC}$ & $700-900-\mathrm{RC}$ & $800-900-\mathrm{RC}$ & $900-900-\mathrm{RC}$ \\
\hline $\boldsymbol{I}_{\mathbf{D}} / \boldsymbol{I}_{\mathbf{G}}$ & 0.83 & 0.93 & 0.92 & 0.93 \\
\hline
\end{tabular}

Table S2 The relative contents of N species of T1-900-RC.

\begin{tabular}{c|cccc}
\hline Samples & $\mathbf{N}_{\text {pyrrolic }}(\%)$ & $\mathbf{N}_{\text {pyridinic }}(\%)$ & $\mathbf{N}_{\text {quaternary }}(\%)$ & $\mathbf{N}_{\text {oxide }}(\%)$ \\
\hline 600-900-RC & 51.2 & 44.7 & 0 & 4.1 \\
700-900-RC & 42.7 & 32.1 & 18.7 & 6.6 \\
800-900-RC & 36.4 & 28.4 & 24.5 & 10.6 \\
900-900-RC & 34.3 & 26.5 & 28.8 & 10.4 \\
900-900-RC-air(20min) & 36.3 & 24.0 & 30.5 & 9.2 \\
900-900-RC-air(1h) & 39.5 & 26.2 & 25.8 & 8.5 \\
$900-900-R C-a i r(3 h)$ & 50.0 & 25.7 & 15.2 & 9.1 \\
\hline
\end{tabular}

Table S3 Surface element composition of T1-900 (results from XPS).

\begin{tabular}{c|ccc}
\hline Samples & C (\%) & N (\%) & O (\%) \\
\hline 600-900-RC & 89.06 & 2.07 & 8.87 \\
700-900-RC & 89.11 & 1.65 & 9.24 \\
$800-900-R C$ & 89.66 & 1.76 & 8.58 \\
900-900-RC & 91.64 & 1.12 & 7.24 \\
$900-900-$ - - air(20min) & 91.31 & 1.16 & 7.54 \\
$900-900-R C-a i r(1 h)$ & 91.27 & 1.14 & 7.58 \\
$900-900-R C-a i r(3 h)$ & 92.20 & 1.10 & 6.70 \\
\hline
\end{tabular}

